\title{
Context-aware Control for Personalized Multiparty Sessions in Mobile Multihomed Systems
}

\author{
Jose Simoes \\ Fraunhofer FOKUS \\ Kaiserin-Augusta-Allee, 31 \\ 10589 Berlin, Germany \\ $+493034637218$ \\ jose.simoes@fokus.fraunhofer.de
}

\author{
Christophe Janneteau \\ CEA LIST, \\ Laboratoire des Systèmes \\ Communicants, Point Courrier 94, \\ Gif sur Yvette, F91191 France \\ +33169089182 \\ Christophe.Janneteau@cea.fr
}

\author{
1 Susana Sargento \\ 1 Universidade de Aveiro, \\ Instituto de Telecomunicações \\ Campus Universitário de Santiago \\ 3810-193 Aveiro, Portugal \\ +351234377900 \\ susana@ua.pt
}

Nuno Carapeto

Portugal Telecom Inovação, SA

Rua Eng José Ferreira Pinto Basto

3810-106, Aveiro - Portugal +35169089182

nuno-f-carapeto@ptinovacao.pt

\author{
Josephina Antoniou \\ Christophoros Christophorou \\ University of Cyprus \\ 75 Kallipoleos Street \\ CY-1678, Nicosia, Cyprus \\ +35722892686
}

\{Josephin,christophoros\}@cs.ucy.ac.cy

\author{
12 Augusto Neto \\ 2 Instituto de Informática, Universidade \\ Federal de Goiás \\ Campus II, Bloco IMF I, Sala 239 \\ 74001-970, Goiânia, Brazil \\ +556235211181 \\ augusto@inf.ufg.br
}

\begin{abstract}
Telecommunication and Internet services are constantly subject to changes, seeking customer's satisfaction. Enriching services with innovative approaches such as context-aware, mobile, adaptable and interactive mechanisms, enables users to experience personalized services seamlessly across different technologies. Aiming at evolving mobile multimedia multicasting to exploit the increasing integration of mobile devices with our everyday physical world, a context-aware multiparty delivery needs to research into two important frameworks: context detection and distribution and context-aware multiparty networking, encompassing adaptations at the session, transport, and network layers. The paper diverges into the second by focusing on the user perceived Quality of Experience and efficient network support of real-time group communications, allowing dynamic adaptation of the multiparty delivery, group communications optimization and maximization of group member's overall satisfaction.
\end{abstract}

\section{Keywords}

Context-awareness, Mobility, Multicasting, Multiparty, Personalization, QoE, QoS, Session Management

\section{INTRODUCTION}

The current technological trend towards context-aware, adaptive systems has brought new requirements in the telecommunication industry for effective usage of context and support of adaptive, context-aware content casting. Context (e.g. network condition, environmental/user information) can be used intelligently to achieve satisfactory user experience and economical use of resources. Context-aware applications and networks should react to context changes to take more efficient decisions according to the environment, session, user and network characteristics. For instance, on the session side, mobile terminals may automatically switch from ringing to vibrating mode inside noisy environments (e.g., mass events) or places requiring silence (e.g., theatres); on the mobility side, the co-existence of multiple Radio Access Technologies (RATs), could be utilized to enhance user experience by recognizing changing context (e.g. a deterioration of network condition) and seamlessly transferring an ongoing session between RATs; on the QoS side, efficient re-routing can be deployed when changes in network/user context is detected, such as increasing on losses and delays or terminal handovers.

Our approach, developed in the framework of the European Project C-Cast [1], aims to support personalized session content delivery to multiple mobile users, independently of the underlying network access and transport technologies, taking context information into account to optimize the content delivery. In the proposed approach, dynamic events based on context changes can trigger session and network reactions, such as service and network reconfiguration, multiparty session content delivery, renegotiation, and seamless context-aware mobility. These reactions need to be efficiently handled by the architecture, in order to increase scalability in such a constant changing environment. We propose an architecture composed by well-defined components that support context-aware multiparty session and networking, which is able to perform the required adaptations at the session, transport, and network levels, triggered by context changes.

The paper is organized as follows. Section 2 describes related work and how it relates to the proposed approach. Section 3 presents the architecture, its components and functionalities and important implementation decisions. Section 4 depicts the most important use cases that illustrate how the architecture works. Finally, section 5 concludes the paper and addresses future work.

Permission to make digital or hard copies of all or part of this work for personal or classroom use is granted without fee provided that copies are not made or distributed for profit or commercial advantage and that copies bear this notice and the full citation on the first page. To copy otherwise, or republish, to post on servers or to redistribute to lists, requires prior specific permission and/or a fee. Mobimedia'09, September 7-9, 2009, London, UK. Copyright 2009 ICST 978-963-9799-62-2/00/0004 ...\$5.00 


\section{RELATED WORK}

Personalized sessions can be influenced by varying context, thus, allowing users access to sessions based on their indoor location, preferences, profile and capabilities [2]. Today, different communication behaviours such as user-initiated communication mediated by the network, situation-oriented and network-initiated communication mediated by the environment, will be possible, requiring the concept of smart spaces to become an integral part of global group communications. In this sense, we aim at extending the smart space concept in the heterogeneous networking environment to provide ubiquitous computing, and support of dynamic group management and personalization.

In next generation networks, multiple access networks will coexist on a common service platform. Consequently, an access selection process, using any-constraint algorithm based on context, preferences and capabilities, should be in place to enable the optimization of both terminal and network [3]. Although many proposals base the decision process on radio signal properties (e.g. [4]), this is only one of the many criteria in a network selection scheme. Thus, some proposals suggest that selection decisions should be context-aware [3]. Moreover, the majority of related work focuses entirely on network selection algorithms, not concerning other important mechanisms crucial to support the decisions made by mobility protocols and QoS management, to enable the complete network re-configuration triggered by context. This lack of high-level perspective is addressed in more recent proposals, [5]. We consider the support of context-aware selection, in a multicast environment, where the group membership is a main issue, still making it flexible enough to support any parameter envisioned.

In fact, literature review shows the lack of current standard solutions to deal with multihomed scenarios. The Stream Control Transmission Protocol (SCTP) [6] has limitations in supporting simultaneous communication on different interfaces, reordering and load balancing, as well as it is not able to provide information on best path selection. The Host Identity Protocol (HIP) [7] evolves towards mobility management without support about technologies and QoS attributes of local network interface. Objective [8] and Profit [9] function mechanisms are not transparent to the users, since they ask data user for decisions. Consumer surplus [10] on the other hand uses a user-centric approach, which may not be good for load balancing of the whole network. Stochastic programming approach [11] is designed for supporting a single common service with fixed required bandwidth, which is not appropriate to a variety of services along with various bandwidth requirements.

Concerning QoS, the integrated deployment of class-based QoS and IP multicast is promising, since the former allows a scalable QoS approach while the latter saves bandwidth [12]. However, this integration is not trivial [13], for instance while QoS achieves scalability by pushing unavoidable complexity to edge routers, IP multicast operates on a per-flow basis throughout the network. Furthermore, the dynamic addition of new group members may affect existing traffic [14]. To overcome these issues, the solution in [15] deploys a coupled control of QoS and multicast resources.

In order to support a variety of multiparty applications in a heterogeneous networking environment with changing networking and environmental contexts, a generic multiparty transport overlay (MTO) needs to be considered. Indeed, mechanisms available in today's networks are using some form of IP tunnelling (e.g.
MBMS [16] or AMT [17]) or specify application layer protocols [18] [19] to provide partial solutions targeted at specific deployment scenarios. As there is no generic multiparty transport overlay able to support hybrid delivery (IP unicast and IP multicast) of multiparty application data, with dynamic management of multiparty transport connections, (i.e., dynamic unicast/multicast switching support, creation/update/ removal of multiparty transport connections), which is simultaneously application-layer independent, the proposed multiparty delivery system will address these issues.

On the session layer, most of the solutions proposed use the Session Initiation Protocol (SIP) as main signalling protocol on IP networks. It has been used in MPLS-based next generation networks [20], addressing point-to-multipoint session management schemes as an enabler for session mobility in converged networks [21]. Considering terminal mobility, paper [22] describes a framework for managing connections to mobile hosts in the Internet. The framework integrates quality of service management and mobility management as the basis for overall session management. Enabling session management mechanisms with context-aware information, the approach of [23] exploits strategies involving the use of contextual information, strong process migration, context-sensitive binding, and location agnostic communication protocols for "follow-me" sessions. Although interesting, these do not cover QoS and efficient multiparty delivery systems.

Finally, much effort has been done recently on the cognitive network concepts [24][25], where cognitive processes can perceive network conditions, and plan, decide, and act on these conditions. It can learn from the impact of former adaptations and accordingly make future decisions, while considering end-to-end goals. Cognitive networks are promising for wireless networks, which are highly dynamic and complex to manage. Our approach is towards the cognitive concept by enabling the dynamic optimization of the use of the network taking into account also the context of the users, sessions and environment.

Concerning standardization, there are several initiatives in the area of multimedia, multicast and broadcast, such as TISPAN architecture and the IP Multimedia Subsystem (IMS) [26], Multimedia Broadcast Multicast Services (MBMS) [27] and Digital Video Broadcasting (DVB) [28]. Our architecture will take into account these developments and will be built upon them, enhancing them with context-aware optimized multi-party delivery.

\section{CONCEPT AND ARCHITECTURE 3.1 Architectural Overview}

In order to understand the real purpose of our paper, it is necessary to contextualize our work into what is being done in parallel to achieve the goals of the C-CAST [1] (Context Casting) project. In this sense, figure 1 depicts the reference architecture of C-CAST, whose main strategic objective is to evolve mobile multicasting to exploit the increasing integration of mobile devices with our everyday physical world and environments.

In order to provide an end-to-end context-aware communication framework specifically for intelligent multicast-broadcast services, three key issues need to be addressed: 
- Development of context and group management service enablers for context representation, context assisted group management and context reasoning.

- Definition of a framework to collect sensor data, distribute context information and manage efficiently context aware multiparty and multicast transport.

- Development of mechanisms for autonomous context driven content creation, adaptation and media delivery.

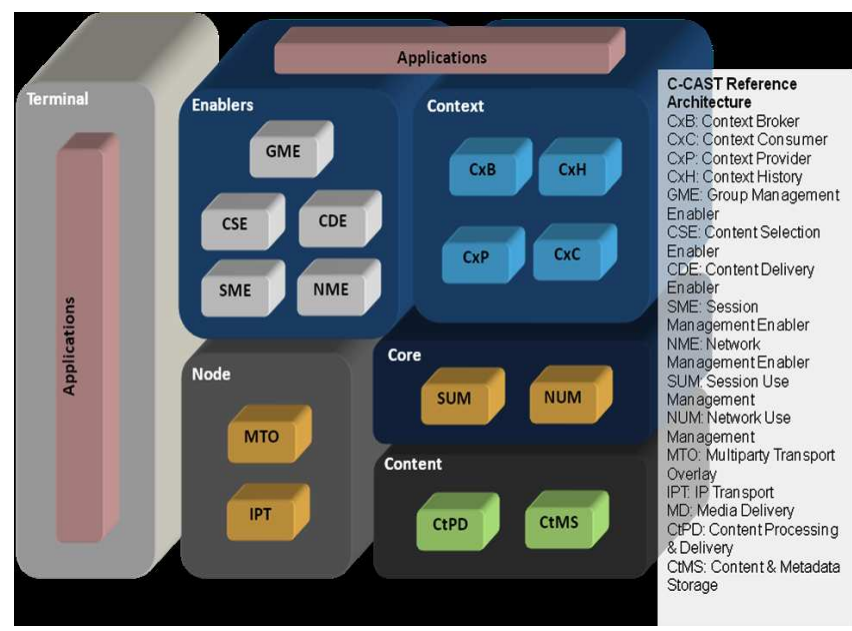

Figure 1. C-CAST Reference Architecture.

From figure 1 it is easy to identify that the architecture is separated into different but complementary functionalities: Context, Content, Core, Node and Enablers. Our work will mainly focus on the last three,

\subsection{Functional Overview}

In the previous section we introduced the main entities involved in the overall content to context architecture; however, it is important to know how each one of them work and how they communicate with each other. Figure 2 details the functional architecture of C-CAST, focusing only on the multiparty delivery layers, where our contributions are most notable. To simplify, in this section we abstract the content to context mechanisms as well as initial grouping and application algorithms, as they are out of the scope of this paper.

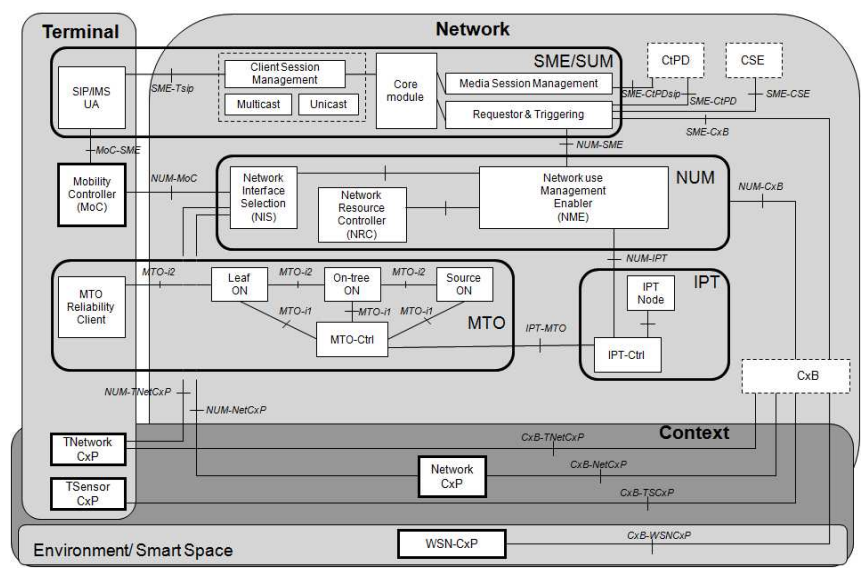

Figure 2. Context-aware Multiparty Delivery Architecture.
In a nutshell, figure 2 includes the following components:

- SME/SUM: The Session Management Enabler/Session Use Management component handles context-aware session control of a multiparty session, more specifically its initiation, modification/renegotiation and termination.

- NME/NUM: The Network use Management Enabler/Network Use Management component is in charge of resource-related selections and decisions, including selecting the best multiparty delivery path towards group members.

- MoC: The Mobility Controller component is in charge of applying the decision issued from the Network Interface Selection (NIS) sub module of the NME/NUM, related to the network interface to be used in the terminal.

- IPT: The IP Transport component is in charge of enforcing the routing path and QoS in the network for the multiparty session.

- MTO: The Multiparty Transport Overlay component is in charge of enforcing the creation, update and removal of overlay trees in the network, providing a generic, scalable, and efficient transport service for group communications by applying the overlay paradigm at the transport layer.

- TNeworkCxP: The Terminal Network Context Provider provides the terminal's view of user networking context.

- WirelessAccessCxP: The Wireless Access Context Provider provides context information from the wireless part of the network.

- NetworkQoSCxP: The Network QoS Context Provider provides detailed information about the current network QoS characteristics. More specifically, it will provide QoS information related to any specific path between two different network nodes.

- TSensorCxP: The Terminal Sensor Context Provider provides the terminal's view of user environmental context, acquired from terminal sensors.

- WSN-CxP: The Wireless Sensor Networks Context Provider provides environmental context at specific areas (e.g. smart places), acquired from locally deployed wireless sensor networks.

These components, as well as the interactions between them, will be described in detail in the following sections. All together, they allow (a) making networking and environmental contexts available and (b) using this context information to drive the following adaptations for context-aware multiparty delivery:

- Adaptation in access network selection, including (a) context-aware RAT selection for mobile terminals aiming at enhanced network capacity and performance, and (b) 802.21 and SIP-based Terminal Mobility between accesses triggered by network or terminal.

- Adaptation in routing path selection, consisting in selecting the best multiparty delivery path based on network characteristics (bandwidth, load, delay, jitter, loss rate, multicast capabilities, etc.) and QoS needs from the session. 
- Adaptation in media coding selection (called subgrouping), consisting in efficient and scalable selection of best media coding per sub-groups of users based on available content formats, device capabilities (e.g. codecs, resolutions supported), user preferences, and network QoS capabilities.

- Adaptation in user device selection, with context-aware SIP-based User Session Mobility between terminals triggered by network or terminal.

- Adaptation in transport connection type, based on a Multiparty Transport Overlay (MTO) providing an efficient transport service for group communications by hiding heterogeneity of underlying networks in terms of IP multicast capabilities or IPv4/v6 support. MTO tree configuration and update is driven by characteristics of multiparty delivery paths to receivers.

- Adaptation in transport reliability, through an adaptive FEC-based reliable transport service well suited for streaming services with stringent latency constraints.

- Adaptation in QoS support, through dynamic QoS enforcement along the multiparty delivery tree (with unicast and multicast branches).

\subsubsection{Context Providers}

In order to feed the architecture with context-aware information, we need entities that are capable to obtain basic contexts from sensors and networks, provide this information in an interpretable manner and deliver this information, making it available to other components. Such entities are called Context Providers (CxP) and they are capable to adapt or report sensed and network information according to control mechanisms, triggering events on other components (using notify/subscription mechanisms).

However, these are not only for applications in the terminal, but also for network and content adaptation. Furthermore, the context information collected by cell phones' embedded sensors can be still enriched by the information obtainable from Wireless Sensor Networks (WSN) deployed in smart spaces. The context data supplied by these WSN would help covering the gaps imposed by cell phone limitations (e.g. situation recognition, microclimate status and indoor location) as well as providing information that would be difficult to be captured by cell phone sensors, e.g., mood or health status. Although, there are already several industrial standards available, which aim at unifying sensor interfaces and data formats, this is not yet achieved in a context-aware multiparty networking framework. The requirements for the capabilities of the sensors in terms of resolution, accuracy and response time depend on the various kinds of context information and may be influenced via a specific module within the C-CAST architecture, user profiles, settings and terminal parameters.

\subsubsection{IP Transport}

The IP Transport (IPT) is responsible to control the integrated QoS and IP multicast enforcement in the nodes along a communication path for the efficient delivery of multiparty sessions to groups of users, with QoS-guaranteed over time. The performance limitations of existing/standard proposals (mainly based on per-flow control) motivated the design of IPT's operations to take into account performance and efficiency. Therefore, main requirements of IPT include the support of: 1) integration of QoS and IP multicast and unicast control; 2) setup of network resources; 3) QoS mapping. IPT handles network resources aggregately received (e.g., in a per-class basis) through information received from the NUM/NME, to overcome the performance shortcomings of existing per-flow approaches.

QoS control is dynamically done, in different QoS models, by means of per-class resource reservations from source to destinations (requested per-flow). For multiparty transport, Source Specific Multicast (SSM) [29] is adopted, being IPT the responsible to correctly populate the Multicast Routing Information Base (MRIB), to allow QoS-aware IP multicast over asymmetric environments (legacy IP multicast lacks QoS support and access control, which is essential in multimedia environments). IPT must support seamless resilience, aiming at attempting to reconfigure multicast trees without changing the current IP multicast address and preventing additional user resubscriptions. The benefits of such process include reduction in terminal energy consumption, in signalling overhead, and in session disruption during handovers due to processing, as well as improvement of user satisfaction.

In order to achieve scalability, resource allocations are coordinated by network edges, thus interior routers remain simple by reacting upon signalling, network events (e.g., link failures, rerouting or mobility) or context changes. As input, IPT takes session context and collects network context directly from devices or QoS-CPs (CPs with QoS information about nodes, paths, etc.) via a well-defined API. IPT is prepared to interact with elements (packet scheduling mechanisms, QoS approaches for mapping, unicast/multicast routing protocols, etc.) of different network technologies to deploy resource allocations and build delivery trees in heterogeneous environments.

The IPT global module can be separated into two different submodules, as shown in figure 3. First, attached to the NUM/NME in the high-level plan, similarly to a plug-in, exists the IPT Controller, which acts as a master component. Furthermore, in the low-level plan, there are several IPTs components placed inside the network acting as slave components receiving primitives from the IPT Controller in order to enforce all the policies decided for each specific IPT Node.

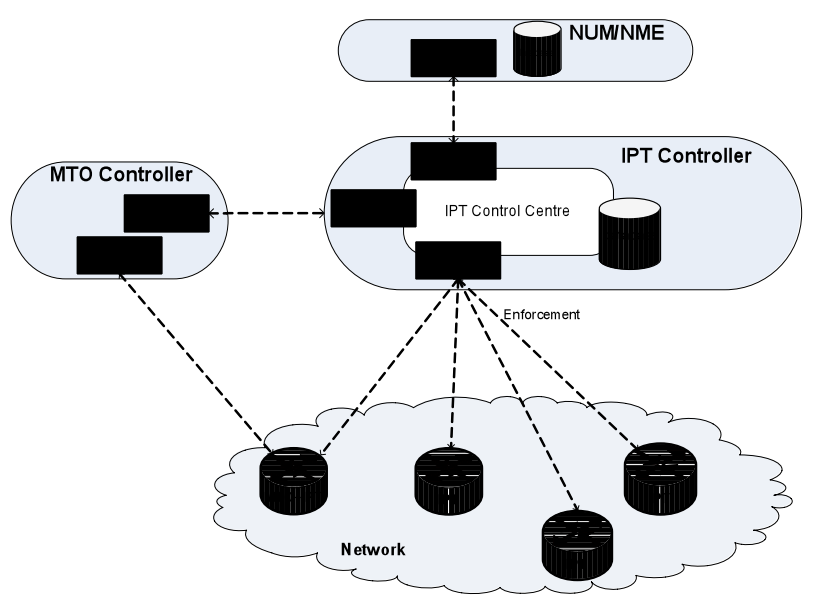

Figure 3. IPT global internal architecture. 
The whole scheme of operation and interaction of these elements is based in a hierarchal model. The final decisions come always from the high-level plan (NUM/NME and IPT Controller) and are passed to the low level plan (IPT Nodes) whose priority is to try to implement the requests received from the upper layer, such as an user sending an IPv4 IGMP request message to join a multicast group. This request is sent by the low level element to the IPT Controller and it is forwarded to the NME through NUM. After this element decided the appropriate path and QoS policies for this user, the request is sent to the IPT Controller responsible for enforcing the several IPT Nodes that will be involved in this specific session for this user.

Besides the interaction to enforce policies in the IPT Nodes, the IPT Controller interacts with NUM/NME receiving requests to provide information about the IPT Nodes. All this information can be stored in a table present in the internal database of the NUM module containing the network topology. This table will be updated with the feedback provided by the IPT Controller responses based on information provided by IPT Node elements. The type of information can include general aspects about the network interfaces associated to each IPT Controller. The IPT Node element is responsible for implementing/enforcing the QoS requests in the network plan. The approach chosen to implement QoS model is based on Differentiated Services (DiffServ). This mechanism for traffic management is class-based and operates on the principle of traffic classification, where each data packet is classified according to their priority and placed in a differentiated row of attendance. For instance, real time traffic should have higher priority compared to non real time traffic. One relevant aspect is that the MTO+IPT node interacts with the IPT Controller module that should be able to behave as a parser between the MTO-NUM requirements.

\subsubsection{Multiparty Transport Overlay}

The Multiparty Transport Overlay (MTO) is in charge of providing a generic, scalable, and efficient transport service for group communications by applying the overlay paradigm at the transport layer. MTO allows hiding the heterogeneity of underlying networks in terms of IP multicast capabilities or IPv4/v6 support, thus allowing any user to participate in a multiparty delivery session irrespective of the network he/she is attached to and in a transparent manner to the application. MTO enables the dynamic creation of an overlay tree, composed of Overlay Nodes $(\mathrm{ON})$, between the source and the group members. The branches of the MTO tree are made of transport connections (unicast or multicast) over which the $\mathrm{ON}$ forwards the content to be delivered towards the group members.

There are three types of ONs; all controlled by a fourth entity called the MTO Controller:

- Source Overlay Node (S-ON): it represents the functional component of the MTO tree that receives the application data flow directly from the source.

- Leaf Overlay Node (L-ON): it represents the functional component of the MTO tree that is the last hop ON from the source to the receiver.

- On-tree Overlay Node $(O-O N)$ : it represents the functional component of the MTO tree that is on the path between the $\mathrm{S}-\mathrm{ON}$ and L-ON.

- MTO Controller (MTO-Ctrl): it represents the functional component of the MTO tree which is in charge of generating port numbers of multiparty transport connections of the MTO tree and pushing them to the IPT as well as to the different ONs of the MTO tree. The MTO-Ctrl also interacts with the IPT module to manage the MTO tree (creation, update, and deletion).

The branches of the MTO tree are made of transport connections (identified by a unicast source address and port, and a unicast/multicast destination address and port) over which overlay nodes forward the content to be delivered towards the group members. Each $\mathrm{ON}$ is forwarding content from one incoming transport connection to one or multiple transport connections based on its own forwarding table. One MTO tree is dynamically established to support one particular multiparty delivery session.

The MTO controller is the entity in charge of enforcing the creation/update/removal of the MTO tree in the network. This basically consists in pushing to each ON of an MTO tree its forwarding table. The MTO controller also interfaces with the IPT component to obtain the list of ONs to form the MTO tree as well as to obtain the IP multicast addresses for the multicast branches of the MTO tree. The MTO controller will complement this description of the MTO tree by assigning ports for all connections of the overlay, and will enforce this MTO tree by pushing the corresponding forwarding tables to each identified ON. The MTO will also return this complete description of the MTO tree to the IPT, allowing the latter 1) to enforce QoS on the multiparty delivery path (identified by the transport connections of the MTO tree) and 2) to interface with the NUM to pass to SME the transport connection information to be announced to the group members. Of course, reconfiguration of the MTO tree is handled by the MTO controller, upon request from the IPT, to accommodate mobility of a group member between access networks requiring a change in the transport connection supporting the multiparty delivery. An example of such a case is when a user moves from an IPv6-only multicast-capable access (e.g. Wi-Fi) to an IPv4-only unicast-only capable access (e.g. GPRS).

\subsubsection{Network Management}

The Network Management Enabler (NME) and Network Use Management (NUM) functional entity is the element that provides intelligent network selections according to the user, network, session and environment context, allowing terminals to be always best connected and to be able to receive multiparty content with QoS guaranteed. The context information is used to settle on intelligent network selection in terms of terminal interface, access technology and communication path. The NME/NUM is designed to work properly on very dynamic scenarios where a large set of network events (link failures, handovers and traffic conditions) take place randomly. Network selection is essential since it is assumed that terminals will contain different, simultaneously available access technologies (e.g., Wi-Fi, WiMax, UMTS, GPRS, etc) with overlapping coverage areas. Therefore, NME/NUM makes use of: user, environment, network and session context, to drive intelligent network selection, in terms of communication path, terminal interface and access technologies. Whereas user, environment and network context can be retrieved in the Context Providers (CPs) by means of the Context Broker (CB), session context is derived from the SME/SUM. Moreover, it is expected to achieve more efficient resource utilization, as well as more uniform distribution of data load, while fulfilling the QoS required by sessions and by the users. For instance, the Wi-Fi 
incoming interface of a terminal should be changed to the WiMax during congestion periods, or after terminal mobility to an area without Wi-Fi coverage area.

It is specified as a modular approach to allow flexible deployment of dynamic operations. Figure 4 depicts the overall architecture of NME/NUM and its main interactions. NME/NUM encompasses two main functional entities: Network Management Enabler (NME) and Network Use Management (NUM).

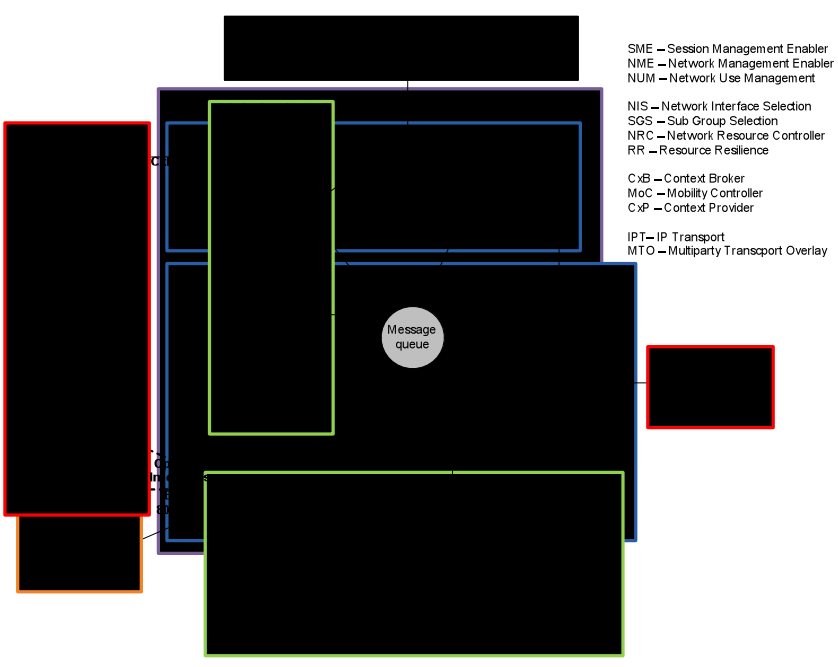

Figure 4. Detailed Network Management architecture.

The Network Management Enabler (NME) is the NME/NUM module responsible for the group management at network level. It splits the service groups into several network-based sub-groups according to user, network, operator policies and a large set of context information. NME comprises the Sub-Group Selection (SGS), the Resource Resilience (RR) sub component and the set of different interfaces.

\section{Sub-Group Selection (SGS)}

The Sub-Grouping Selection (SGS) receives requests from the SME/SUM for resource setup, modification and teardown for session groups, i.e., service groups refined according to content formats availabilities matched with terminal capabilities. Once the SGS receives a request, it triggers the network selection process in order to perform an intelligent radio access selection. Furthermore, it requests a communication path selection, multicast or unicast, with specific quality requirements. Based on the network selection and chosen communication path, the SGS organizes user subgroups, which are finally refined taking into account context information. SGS has its own logic for sub-groups creation, but it can be evolved easily with new algorithms or methods to improve the system performance. Furthermore, it can be extended easily to cope with new set of context information leading to refined subgrouping creation. Once the sub-groups are created and properly enforced, this information is returned to the SME/SUM entity with the respective addresses and ports and selected coding for each sub-group.

\section{$\underline{\text { Resource Resilience (RR) }}$}

The Resource Resilience (RR) subcomponent is a part of the NME sub module, connects with NUM topology databases, and supports NetworksQoSCxP interface. Currently it has two main tasks: interacting with the $\mathrm{CxP}$, filling and maintaining updated the topology database, both at start-up and periodically during the operation; and proving NetworksQoSCxP the information about ongoing sessions to be able to verify if the intended QoS is maintained. For this reason, it may trigger sub-grouping updates in the SGS.

The Network Use Management (NUM) is the NME/NUM module responsible to manage network resources allocation. It encompasses the Network Interface Selection (NIS) aiming at providing intelligent radio access technology selection based on context-aware information to groups of users. NUM also includes the Network Resource Controller (NRC) module, which is in charge for the communication path selection based on network information.

\section{Core Broker}

The Core Broker is in fact a message router or BUS that forwards information and triggers between the different modules of both NME and NUM. It maintains the consistency and provides interfaces for the different sub modules between modules. All the described components act as a plug-in of the broker and can be added or removed during runtime.

\section{Network Interface Selection (NIS)}

The NIS is a sub-component in NUM suite that aims at providing intelligent context-aware RAT selection to the users belonging to a group, both at the session initiation and during the session duration. The intelligence context-aware RAT selection algorithm running in NIS is taking into consideration

- the instant networking and environment context of the users belonging to a group (RATs/Cells/Access Points within the Terminals' reach, pilot channel quality received, pilot channel quality alteration rate experienced, speed, location, etc.),

- the whole heterogeneous network context conditions (current load in RATs, available capacity and current load in Cells/Access Point belonging to each RAT, RAT's capabilities and QoS that can be supported by each RAT, etc.),

- the profile of the users (Priority of the user, Network preferences of the user, User's Terminal capabilities, etc.),

- some rules/constrains that should be obeyed within the network during the multicast content provision in order to avoid any undesirable events predicted by NIS to occur in a short time,

estimates all "possible" and "acceptable" transmission arrangements (i.e. different combination of which RAT will support each user and the content coding quality that each user will receive) that can be used to distribute the multiparty session content to the users and selects among them, the most efficient one. The rules/constrains referred above are defined dynamically by the NIS by considering both the instantaneous and the history context information of the network and users collected through time. For example, previously received context is compared with 
current context for similar patterns to be located. Based on the similar patterns located, using some intelligence, NIS forecasts undesirable events that are possible (e.g. congestion in the Network, overloading of certain RATs, users' QoS degradation, etc.) to happen and sets some rules/constrains that should be obeyed during transmission arrangement selection in order for these events to be avoided. An additional parameter that could be considered to influence the decision of RAT selection is possible traffic forecasts over certain time periods in a multi-Network Operator settings. Such demand forecast should be assigned fairly between different Network Operators and considered during the RAT selection decision.

After deciding the transmission arrangement that will be used, NIS informs each Terminal about the selected interface from which multiparty content will be provided (i.e. Wi-Fi, WiMAX, UMTS, LTE, DVB, etc.) and triggers NME to control the MTO trees. NIS will also provide support during the multiparty sessions transport, in which users of on-going sessions can receive multiparty content in a different network interfaces, which can be happen in the event of changing network conditions or even handovers (with support of the mobility controller).

\section{Network Resource Controller (NRC)}

NRC is the NUM module responsible for selecting the best communication path within the network. It receives requests from the SGS to decide on the best path for the multiparty connection. To do that, NRC maps the QoS requirement of the multiparty session into an available class of service, also taking into account all the network status when selecting the path. In this sense, NRC deploys admission control operations along the network communication path. After selecting the appropriated channels for multiparty data transmission, it returns them to the SGS, which finally takes the decision of enforcing the reservation.

\section{Access Network database \& Handover Policies database}

The Access Network database and Handover Policies database includes information about the access networks that are used by NIS and NME, plus all the information related with the IEEE 802.21 including the policies for performing and triggering handover. It acts as a Media Independent Information Service (MIIS).

\section{Network Topology database}

This database includes all the information about network topology that is filled in by NetworksQoSCxP. It has detailed information about the network topology and the individual QoS limitations of each individual link. It provides information to NRC and allows it to build possible multicast paths and enable QoS to the users.

\subsubsection{Session Management}

The Session Management Enabler/Session Use Management (SME/SUM) is an entity found in the Core Network, whose main task is to manage user-to-content and content-to-user relationships within multiparty sessions. SME/SUM is responsible for session control, more specifically initiation, modification/renegotiation and termination of the session. It is intelligently designed to enable the use of context information for session control (using $\mathrm{SIP}$ ), in terms of network-specific, environment-specific and userspecific contexts, without in fact knowing the actual network, environment or service details. The main challenge of this component is to enable the support of multiparty sessions that can effectively adapt to context changes, improving the overall user experience.

In terms of functionality, the SME is the session management module that accepts context information through requests or triggers and responds by creating/modifying/terminating the sessions corresponding to the defined sub-groups. In other words, it handles the interfaces between:

(a) the $\mathrm{SME}$ and the $\mathrm{CDE}$ (the entity responsible for matching a group context to the appropriate content),

(b) the SME and NME (the entity that knows the network),

(c) the $\mathrm{SME}$ and $\mathrm{CxB}$ (where all context information subsides),

(d) the SME and CtPD (the content processing and delivery entity, which requests and receives the content description for a particular service group from the SME, but also interacts with the SUM),

(e) the SME needs to interact with the SUM (which is the sub-module responsible for handling the SIP-specific tasks of the Session Manager, such as inviting the users and the media delivery function to sessions).

Once the Core Entity of the SME determines the matching between available content formats and user capabilities in terms of supporting content formats, resulting in candidate files per user in the service group, SUM takes over, which is responsible of first inviting the users to a session and to invite the CtPD to deliver the content to these users. Figure 6 illustrates the SME/SUM interfaces with other components.

Moreover, the SME/SUM includes a module on the terminal that allows the terminal to understand SIP/IMS, be able to join a multicast group and a set of modules in the network. The set of sub-modules in the network are divided in two categories: the SME-related modules and the SUM-related modules; intracommunication achieved through appropriate method calls of defined interface web services. The SME module includes the sub-grouping functionality, where the decision on subgroups is made, and the Requestor and Triggering functionality, which is responsible for interfaces with other modules for receiving triggers and sending requests. The SUM module includes the client management functionality (e.g. inviting the user to a new session) and the media management functionality (e.g. inviting the $\mathrm{CtPD}$ to deliver content to a subgroup).

\subsubsection{Mobility Controller}

The Mobility Controller (MoC) sits in the mobile terminal and is in charge of applying the decision issued by the Network Interface Selection (NIS) module of the NUM related to the network interface to be used in the terminal. Therefore, the MoC is involved in any vertical handover decision. The interface between $\mathrm{MoC}$ and NUM leverages the so-called Command Service provided by the IEEE 802.21 Media Independent Handover (MIH) standard [30]. SIP-based mobility is used to handle continuity of ongoing multimedia sessions despite change of interface. Therefore, upon receiving a handover command from NUM, the MoC will configure the new interface and will trigger the SIP User Agent on the terminal to perform SIP re-registration with the new IP address, and to send a SIP re-invite for ongoing session(s).

The basic assumption concerning network interfaces in the terminal is that only one interface is active at a given time. 
Therefore, and given that SIP mobility is considered, any change in the selected interface will involve the following sequence:

1) Receive handover command from NIS module.

2) Activate the new interface (and disable the old one).

3) Get a new IP address for the new interface.

4) Ask the SIP UA to perform SIP re-registration with the new IP address.

5) Ask the SIP UA to send a SIP re-invite with the new IP address for ongoing SIP session(s).

Section 4 provides further details regarding the communication that occurs between the NUM and MoC.

\subsection{Design Principles}

The implementation of the context-aware multiparty delivery presented here has been driven by the following design principles:

- Minimize changes in the user terminal.

- Place intelligence in the network; where most of the networking and environmental context information, needed to drive adaptations in multiparty delivery, is available.

- Maximize reuse of existing standards when applicable.

- Distinguish between context-aware decision making \& enforcement.

- Enable fast adaptation to a context change.

The SME/SUM is the entity that receives the user capabilities from the Context Broker and accordingly matches them with the available content formats received by the content processor. The SME/SUM promotes this matching (a form of preliminary subgrouping) to the NUM, which will further refine the users into QoS-specific sub-groups, based on the paths selected for each user. The decision from the NUM will be received by the SME, invoking Session Use Management (SUM) state, responsible for activating a different session per each sub-group. In fact, SUM is the sub-module responsible for handling the SIP-specific tasks of the Session Manager, such as inviting the users and the media delivery function to sessions. The NUM is the entity that performs best path selection according to the network, user and environment context, optimizing both user QoE and network performance in a heterogeneous technology environment. NUM takes as inputs the context information coded in measurable parameters, and performs a decision of the best network to support the users and its flows. It then selects the ONs and the transport mode (unicast or multicast, IPv4 or IPv6) that best fits the users, sessions and network needs. Note that NUM is able to perform a second level of sub-grouping since, depending on the network ability to serve the several users, it may be required to redistribute them among different technologies able to support the services with different bitrates and QoS. This may require that the users receive the content with different codings, through different flows, and therefore, different groups may be created.

The MTO is in charge of enforcing the creation, update and removal of the MTO tree in the network. The MTO interfaces with the IPT to obtain the list of ONs forming the MTO tree as well as the IP multicast addresses for the multicast branches of the MTO tree. This information obviously depends on the path from the source to each group member as previously selected by the NUM. The MTO will complement this description of the MTO tree by assigning ports for all connections of the overlay, and will enforce this MTO tree. The MTO will also return this complete description of the MTO tree to the IPT, allowing the latter: 1) to enforce QoS on the multiparty delivery path (identified by the transport connections of the MTO tree); and 2) to interface with the NUM to pass to SME the transport connection information to be announced to the group members. The reconfiguration of the MTO tree is handled by the MTO, upon request from the IPT, e.g. to accommodate mobility of a group member between access networks requiring a change in the transport connection supporting the multiparty delivery.

\section{USE CASES}

The following section shows the use cases that describe the main interactions between the several architecture elements in different situations and scenarios..

\subsection{Triggers / Contexts}

To endow networks with the ability to rapidly and correctly adapt to any type of event or change, it is crucial to be aware of the context surrounding the different network elements. The type of information that may be used to select the best access network can be found on different entities and within each at different levels. Given the large variety of possible information sources, a complex and possibly "heavy" context acquisition mechanism is required. Moreover, as a large amount of information may be relevant to the decision procedure, an efficient model and organization scheme must be developed in order to make critical context constantly updated.

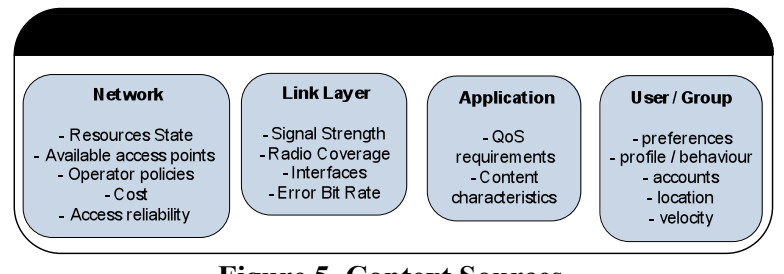

Figure 5. Context Sources.

All this context-awareness encompasses a wide variety of sources and obviously they do not have all equal urgency when any kind of change occurs. Critical context is related with low level constrains such as signal strength, high packet loss rate or delays and lack of resources. High level constraints, as user profile or preferences, may be taken into account in a future access selection decision, as far as the current state of the entity is updated so that when processing the changes noticed they are not outdated. Thus, it is crucial to wisely select which context sources are crucial and deserve immediate attention, and those that do not affect directly connectivity and minimum quality of service. Due to processing time concerns of the selection algorithm, critical context changes are attended on real-time, providing solutions to the network that avoid and prevent service disruption.

\subsection{Grouping / Sub-grouping}

For operators it is crucial that the architecture predicts and reacts promptly to the addition and removal of users. The grouping of users based on desired content is usually done at higher layers, such as the service and application ones. However, due to the variety of context information, the sub-grouping is also performed at Session and Network levels, supported by the session and network context (Figure 6, messages 1 and 13). For example, due to the context information, the best access technologies for the users in the same group may diverge per user, since user context and environment is also taken into account. The different 
networks may have different guarantees, which may require the content to be delivered with different quality and codings, hence requiring users sub-grouping.

Each of the created sub-groups represents a multicast delivery tree or, in case of a single user, a unicast connection, that requires network resources. For some cases, where not all the users or links have the required multicast capabilities, MTO nodes are enabled to abstract the grouping decision of these considerations. Moreover the sub-grouping flexibility allows, for instance, in the same session, two users to receive the same audio, but different video streams with different codecs or rates, just depending on the available resources or preferences. In this case both would be in the same audio sub-group, but in different video sub-groups. To be able to achieve an efficient sub-grouping dynamism, we considered several use cases (see below).

\subsubsection{Session Initiation}

A request arrives at SME and is usually associated with a new group creation. Figure 6 depicts the messaging flows between the different components involved in Session Initiation.

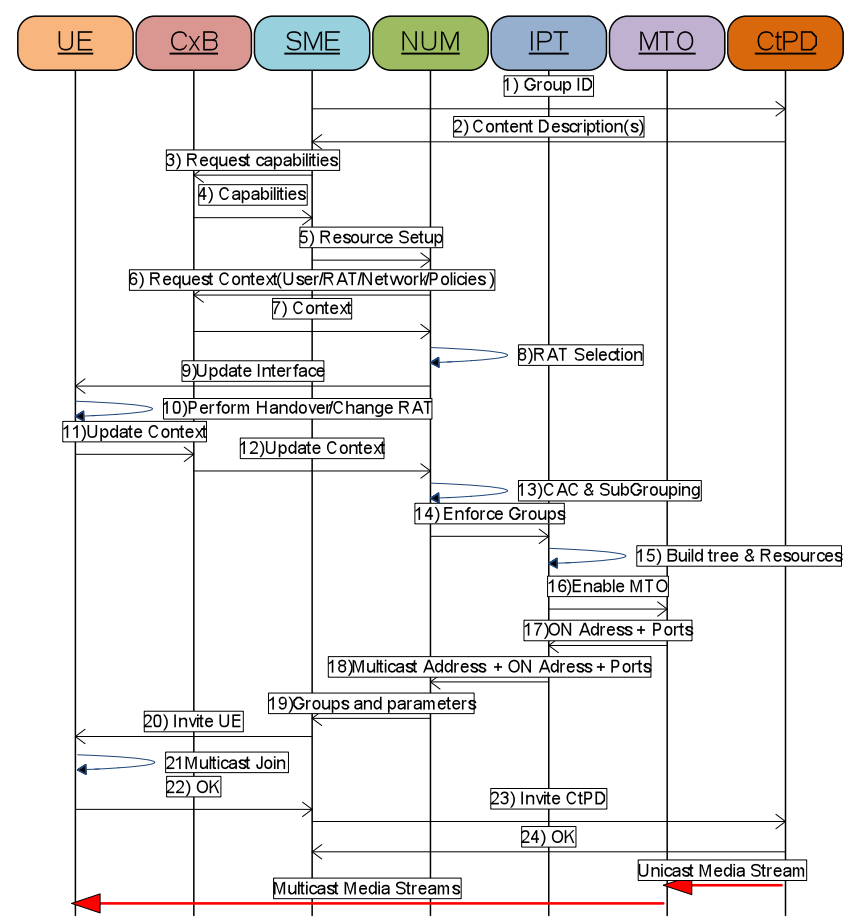

Figure 6. Overview of Session Initiation MSC.

$(1,2)$ - Based on this, the SME requests information about the content to be streamed.

$(3,4)$ - The SME requests information about the user contexts.

(5-12) - This triggers NUM to segment the group in smaller ones considering the operator policies (may prefer the fewer the possible), the characteristics of each terminal, the terminal associated RAT, multicast and QoS capabilities, end to end available resources, etc.
(13-18) - This process might involve network triggered handover to achieve service efficiency. Each sub-group represents a multicast group that is enabled and maintained by IPT and MTO

$(19-22)$ - In the end NME/NUM pushes the SME/SUM to invite all the users.

(23-24) - After, it sends the SIP Invite towards the content provider. Finally, the sessions are established.

\subsubsection{Group Modification}

Comprises the cases where users join and/or leave the group session. This is initiated at SME and propagates through NUM and other modules similarly to session initiation. The removal of users can reduce size or even terminate an existing sub-group. The addition of users is complex and is only considered when the user experience of the new user is not compromised by joining an already streaming context. Figure 7 illustrates the scenario where a user is removed from a group.

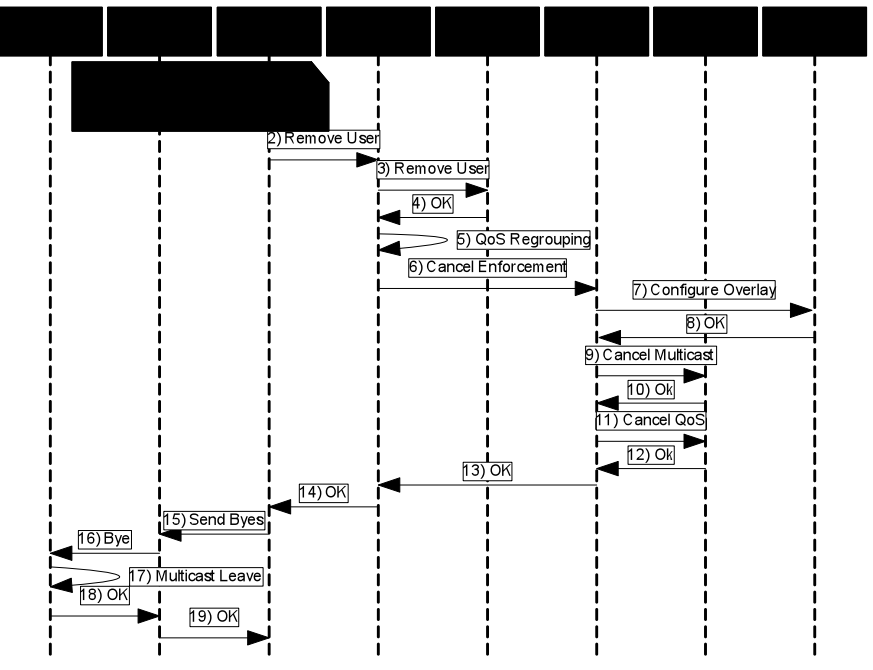

Figure 7. Session Modification when a user is deleted.

(1) - The SME is triggered that a new user is removed from a service group.

(2) - The SME sends a request to the NME to remove the user.

$(3,4)$ - NME asks NRC to remove user, and a confirmation is sent.

(5) - NME performs network level re-grouping.

(6) - NRC triggers the cancelation of the enforcement of resource reservation to IPT controller.

(7) - IPT controller triggers MTO to configure the overlay.

(8) - The confirmation is sent.

(9-13) - The IPT Controller then cancels the multicast tree enforcement and QoS in the nodes, and sends the result to NME.

(14) - The SME receives a confirmation from NME that the user has been removed.

(15) - The SME triggers the SUM to send bye (SIP BYE) to the removed user.

(16) - The SUM sends BYE to the UE. 
(17) - If the UE is multicast-enabled, it performs a leave.

(18) - The UE sends an OK response to the SUM.

(19) - The SUM sends a confirmation message to the SME.

\subsubsection{Sub-Group Modification}

Performed in NUM, refers to those cases where the users are switched between sub-groups. The session group previously created is not modified and only the concerned sub-groups are updated. Whenever this happens, some users get "promoted" (or "demoted") to a group with different quality of service. This can be triggered by the implemented IPT resilience mechanisms, where the network conditions are significantly altered: a link or a router may go offline or back online, the QoS conditions may be altered, the access network may become overloaded, or the terminal may be forced to move into a different network with different conditions. Eventually, session mobility between terminals with different characteristics and updates of operator policies will also trigger sub-group modifications. Figure 8 exemplifies this scenario.

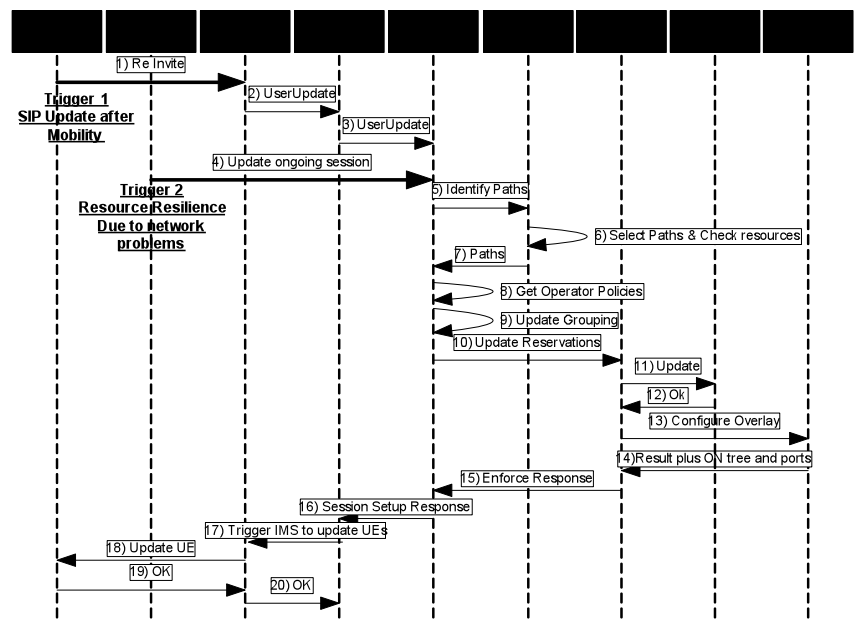

Figure 8. Session Modification User update (sub-group).

(1) - (Trigger 1) After mobility has been performed, the interface triggers one SIP re-invite to SUM.

(2) - The SME is triggered by SUM that a new user has changed interface.

(3) - The SME sends a request to the NME to update the user.

(4) - (Trigger 2) This message represents another possible trigger incoming from the Context Framework (NetworkQoSCxP). It represents problems with ongoing sessions/users and triggers the path update.

(5) - NME sends a trigger to NRC to select new paths that reflect the updates.

$(6,7)$ - NRC selects the best paths according to the network context and resources and sends it to NME.

(8) - NME gets the operator policies in terms of the paths and 9) performs network level sub-grouping according with the chosen paths.

$(10,11,12)$ - Then, NME asks IPT Controller to update the reservations on the IPT nodes and MTO,
(13 -16) - before returning the result to NME and after to SME.

$(17,18)$ - The SME triggers the SUM to update all the affected terminals (SIP Update).

$(19,20)$ - The terminal finishes the SIP signaling and returns OK to the SME.

\subsubsection{Session Termination}

In this case all the users leave the group, or the context that triggered the session changes significantly. NUM frees the resources at IPT and MTO. Figure 9 shows the messages exchanged between the different components involved.

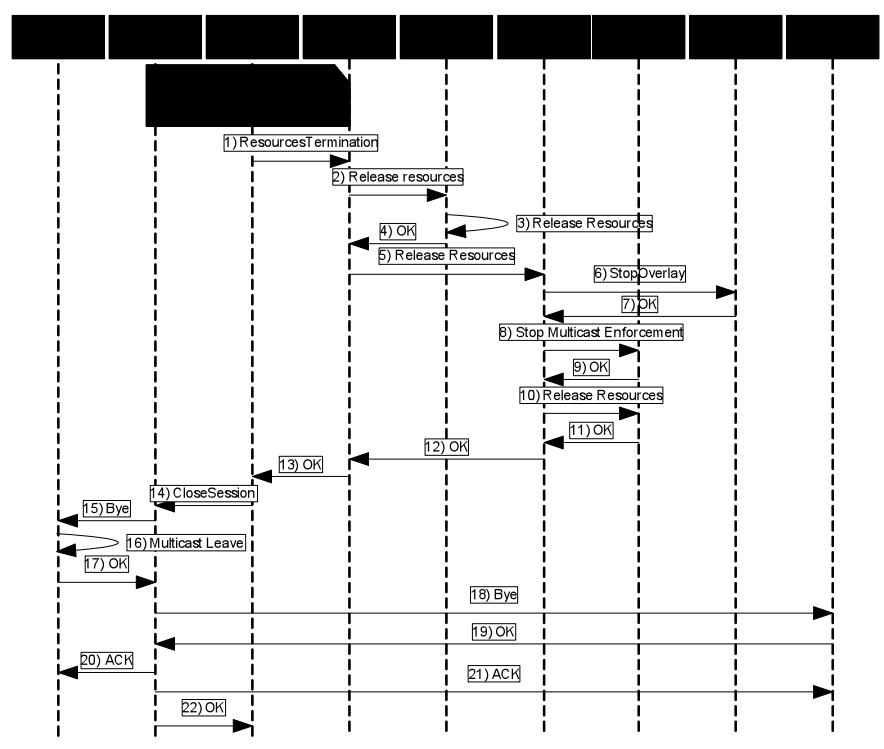

Figure 9. Group Session Termination.

(1) - After the SME is triggered to terminate all the existing sessions for that specific group, the SME (which does not require any external information) requests the NME to release the resource allocation.

(2-4) - NME asks NRC to release resources in this database, the resources are released and a confirmation is sent.

(5) - NME triggers IPT Controller to enforce the resources release in the network.

$(6,7)$ - IPT controller triggers MTO to remove the overlay, and release the overlay nodes from this functionality, whose confirmation is sent.

(8-12) - The IPT Controller then releases the multicast tree previously configured and releases the resources in the nodes, and sends the result to NME.

13) The SME is informed that the resources were unallocated.

14) The SME invoques SUM web service to terminate the group session.

15) The SUM sends a SIP BYE request towards all the end users belonging to that group with currently active sessions.

16) If the user session is using multicast technology, the user sends a LEAVE message. 
17) The user sends a $200 \mathrm{OK}$ message, indicating that he will terminate the session.

18) Once all the users have terminated their sessions (or the timer expires), the SUM terminates the session towards the CtPD interface by sending a SIP BYE message.

19) The CtPD replies with a 200 OK message acknowledging this event.

20) SUM sends ACK towards the CtPD.

21) SUM sends ACK towards the end user to finalize the process.

22) Finally, the SUM informs the SME the all sessions for that specific group have been terminated.

\subsection{Mobility}

To actually guarantee QoS and service continuity during node mobility, the architecture is prepared to select the best RAT and trigger handover mechanisms. This can be done by two ways: either by having the context providers monitoring if the individual parameters from the network instant context go below defined thresholds; or by similarly monitoring different weighted subsets of these parameters plus additional environmental and situational context by the NIS module.

\subsubsection{Triggered Handover}

Both cases of terminal mobility, Terminal Triggered Handover and Network Triggered Handover were considered. Still, independently of the stimulus provision, the process is assisted by the network. NIS takes the decision of selecting the best RAT from the candidates provided by the $\mathrm{CxP}$ and triggers the handover process at the MoC. After the traditional handover mechanisms, the terminal updates its addresses and the providers which through the context framework notify the NUM. Finally the NME checks if is necessary to update the multicast tree, notifies IPT, MTO and sends a session update to the terminal through the SME.

In fact, through time a lot of changes can occur altering the users' and the network's context and also the rules/constrains set by the Intelligence Calculator, possibly making the current transmission arrangement used not acceptable anymore, or even making another transmission arrangement more efficient than the one currently used. Thus NIS, in order to address these variations, must be registered in the CMS to receive periodically (period T) the new users', and network's context. Based on the new context received and the rules/constrains that the new transmission arrangements must obey, identify the new possible and acceptable transmission arrangements and dynamically adapt (by triggering the necessary handovers) to the one considered as the most efficient (in terms of radio resource consumption and network performance). Note that the periodicity of reporting (period T) is a parameter that highly influences efficiency and should be chosen as a tradeoff between the uplink noise/load rise, the Terminal's battery consumption (due to measurement reporting) and the capacity/performance efficiency. For example, the more frequent the measurement reporting the higher the capacity/performance gain but the more the terminal's battery consumption and the more the uplink noise/load rise). Thus, the period $\mathrm{T}$ must be chosen by the Network Operator in such a way that will avoid scarifying the efficiency of the one over the other. Figure 10 exemplifies this process.

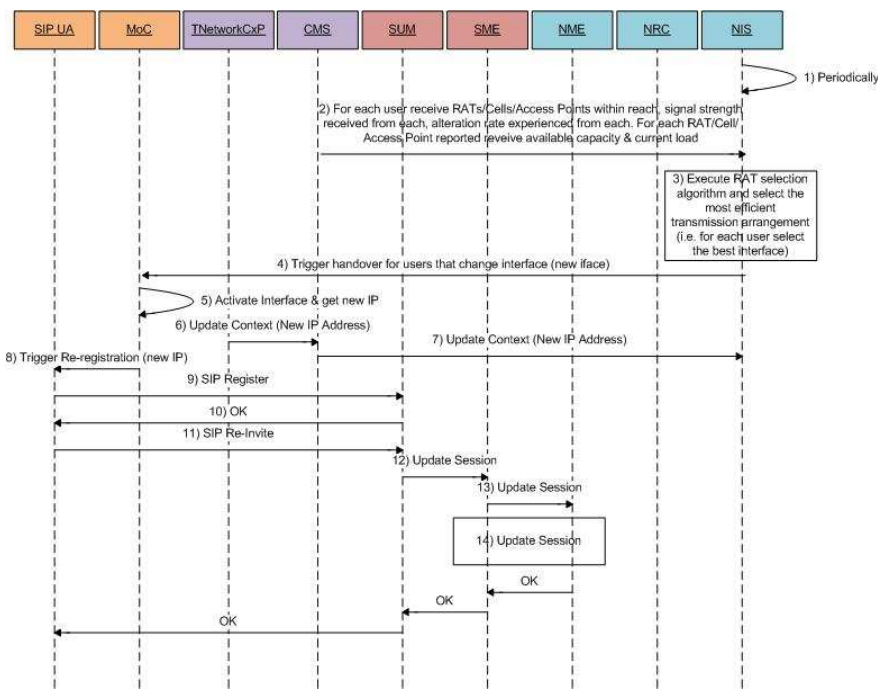

Figure 10. Network triggered handover.

$(1,2)$ - In order to address any change in the context and adapt to the most efficient transmission arrangement through time, the NIS periodically (period $\mathrm{T}$ ) receives from the Context Broker the instantaneous users' and network's context.

(3) - The NIS based on the new context information received, executes the RAT selection algorithm and checks if a more efficient transmission arrangement than the one currently used exists (i.e. checks if the handover of some users between the RATs enhances capacity and performance).

(4) - If a new transmission arrangement is selected, for each user that a new RAT/Cell/Access Point is selected, the NIS triggers the handover of the Terminal to the new RAT/Cell/Access Pont, by sending the new interface selected to the Mobility Controller (MoC) running in the Terminal.

(5) - The Mobility Controller (MoC) then reconfigures the mobile terminal to use the newly selected interface; more precisely it activates the new interface and determines the IP address on that interface.

(6) - The TNetworkCxP detects the new IP Address acquired by the Terminal and updates the CMS (CxB).

(7) - As the NIS had previously registered to TNetworkCxP to receive the user's IP Address whenever a change occurs, the update in 7) results in a notification towards the NIS.

(8) - Then the MoC triggers the SIP User Agent on the mobile terminal (and passes the new IP address) so that the latter triggers the SIP re-registration procedure to the SUM as well as SIP reinvite procedures for all ongoing sessions.

(9) - The SIP UA performs a re-REGISTER in the SIP Registrar server (can be a SIP Proxy, IMS Core or simply an entity associated with the SUM).

(10) - The entity processing the REGISER events replies with a 200 OK message indicating the new registration details.

(11) - The SIP UA sends a re-INVITE towards the SUM in order to update it's address, so that the media is now sent to the new interface.

(12) - The SUM requests the SME to trigger the session update. 
(13) - The SME triggers the session update on the NME (recourse allocation for the new interface).

(14) - The Session is updated.

The following messages are just 200 OK messages acknowledging that the whole process was performed successfully.

\subsubsection{Session Mobility}

On the other hand, Session Mobility, be it or not initiated at the user is considered network assisted. The SME is responsible for evaluating and selecting the best terminal, considering their context, capabilities, user information and content requirements. Such actions will trigger the SIP registration for the new equipment and a NUM update on the multicast tree. These cases have usually a high impact in QoS, given the different terminal capabilities. NUM performs the sub-groups update selecting a different codec and transmission stream. Done with this process, SME moves the session to the new terminal, which joins the multicast group. As an example, during an ongoing multiparty communication, continuity of the service shall be maintained despite of the device being currently used. Like enunciated before, session mobility is the key concept of providing this continuity. In this context, there are two possibilities in which this action might occur: terminal triggered session mobility or network triggered session mobility. The first occurs when a user expresses his/her will to transfer the session from one terminal to another and the reasons for this behavior are numerous (ex. user arrived home and wants to transfer a video session to his high quality television, or wants to go to his/her living room where there is not television available but still can watch the football match in his/her mobile or computer). Network Triggered Session Mobility between devices is depicted in figure 11 .

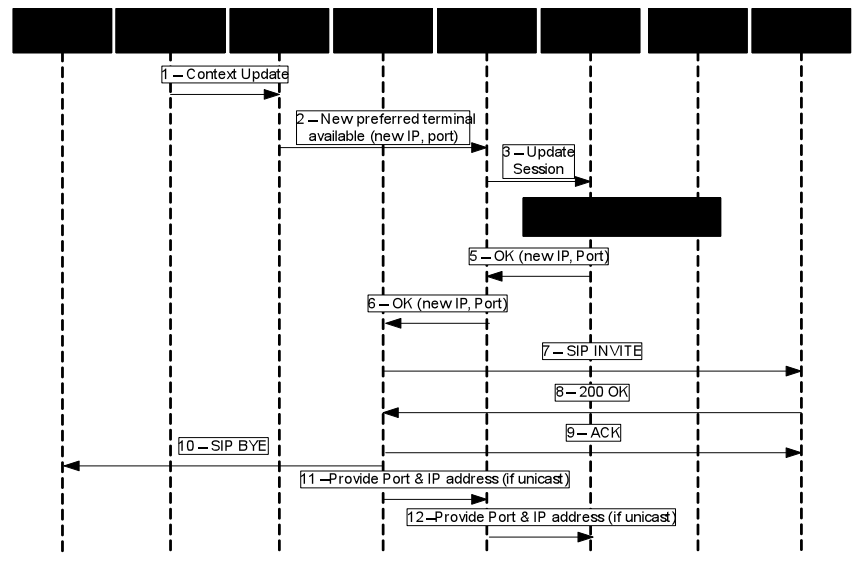

Figure 11. Network Triggered Session Mobility.

(1) - The TNetworkCxP informs the CMS (CxB) that a new context is available. In this situation, it is most likely that a new terminal is available in a determined area.

(2) - When the context broker receives some sensor, location or network update from the context provider installed on the client, it evaluates all the variables and when some criteria are met (some threshold) it might trigger a notification towards the entities that registered for this specific context, in this case, the SME.
(3) - Based on this notification, the SME will perform the session mobility to the preferred device (which is currently not involved in any session). This decision will instruct the SME to update the session, changing the media flows from one terminal to the other. Before contacting the new terminal, the SME contacts the NME to perform bandwidth reservation and allocation to the new destination)

(4) - The NME performs a session update. 5) After processing the resource allocation and all the overlay trees reconfiguration, the NME informs the SME with the new IP and Port were the media will be streamed to the client (in case of unicast) or with the multicast address the new terminal should join.

(6) - This information is then passed to the SUM, indicating that he should establish a session with the new terminal.

(7) - The SUM sends an INVITE to the new terminal with the media settings received from the SME.

(8) - The new client SIP UA replies with a 200 OK message indicating that he is now expecting media and the session can be transferred.

(9) - The SUM acknowledges this message (8) and informs the SIP UA about this.

(10) - The SUM sends a SIP BYE message towards the old terminal (to release the session).

(11) - If the new terminal is only unicast enabled, the SUM informs the SME about the IP and Port where the new user will be expecting to receive the media.

(12) - Then the SME forwards this information to the NME to reconfigure the overlay tree accordingly and stream the media content towards the new terminal.

\subsection{Scalability}

The presented architecture was originally designed with strong concerns for performance. This was addressed in each individual module. The inherent separation of tasks, mechanisms and decision algorithms allow the system to process each update of context independently, consequently only affecting the necessary modules. Events such as the ones described in the following paragraphs do not propagate throughout the system triggering non-essential modifications.

Grouping and sub-grouping is performed mostly to reduce the resources necessary to deliver content and also to reduce the signaling in initiation and update of groups. It allows resources to be managed as a whole and not individually per user. Furthermore the content source does not need to be aware of the quantity, location or network parameters of its clients.

Whenever the RAT is changed, if it is possible and advantageous to maintain the same QoS, the user is kept in the same multicast group. Only the IPT multicast routing tables are affected without even requiring the user to trigger multicast leave/join. If the new RAT implies QoS changes, this will switch the user between multicast sub-groups and force a session update towards the user. Even so, the global session is kept unchanged and the content source does not receive any update. In the eventuality of a network element malfunction or the deterioration of a link QoS level, the IPT implements fast resilience mechanisms. These allow the re-routing of multicast traffic with minimal impact on the endto-end session. This is done by updating the path in-between overlay nodes and keeping the overlay tree. Upper modules do not 
need to be involved and NUM is only notified of updates to keep database consistency.

\section{CONCLUSIONS AND FUTURE WORK}

The use of context allows the definition of mobile communities that are characterized by requiring the same content. The users in similar situations should receive the same data. The mobile network, as well as the multicasting service, should be enhanced to create multiparty sessions that allow content delivery to such groups of users. The context-aware multiparty delivery system proposed in this paper, allows on the one hand making networking and environmental contexts available, and on the other hand leveraging this context information to drive adaptations in multiparty delivery. This is achieved through the support of a dynamic session considering and making use of context-based adaptations including user device selection, access network selection, routing path selection, media coding selection (by subgrouping), transport connection type selection (e.g. unicast/multicast or IPv4/v6), dynamic control of transport reliability, and dynamic QoS enforcement along the multiparty delivery tree. In fact, splitting the content into different media types and delivering it using different sub-groups, allows the architecture to improve the rationale between personalization and efficiency, which is one of the biggest dilemmas operators are nowadays facing.

By early 2010, this architecture will be completely implemented in the C-CAST experimental platform, allowing its validation and demonstration of the value-added capabilities and usages envisaged. In parallel, simulations are also contemplated as a means to assess the scalability and performance (e.g. in terms of adaptation to change in context) of the proposed scheme for context-aware multiparty networking.

Moreover, the solutions presented in this paper are expected to facilitate the emergence of new types of context-aware multiparty applications, which may respond to context changes in order to adapt and satisfy the users of a group through adaptive content delivery. This is enabled by proposed network enhancements resulting in a novel framework that promises to best meet user needs and optimize user experience while enabling efficient use of network resources.

\section{ACKNOWLEDGMENTS}

This work was supported by the European ICT project "C-CAST" - Content Casting - (Contract-No. ICT-216462). The authors would also like to thank the contributions from their colleagues Mounir Kellil (CEA LIST), Pierre Roux (CEA LIST), and all other C-CAST partners for their insightful comments.

\section{REFERENCES}

[1] C-CAST project, web site: http://www.ict-ccast.eu

[2] B. Sigrid, M. Poi, U. Tore, U, "A simple architecture for delivering context information to mobile users", Proc. Work. Infrastructure for Smart Devices - How to Make Ubiquity an Actuality, 2003.

[3] V. Jesus, S. Sargento, and R. L. Aguiar, "Any-constraint personalized network selection", in Personal, Indoor and Mobile Radio Communications, 2008. PIMRC 2008. IEEE 19th International Symposium on, 2008, pp. 1-6.

[4] K. Pahlavan, P. Krishnamurthy, A. Hatami, M. Ylianttila, J. P. Makela, R. Pichna, and J. Vallstron, "Handoff in hybrid mobile data networks," Personal Communications, IEEE, vol. 7, pp. 34-47, 2000.

[5] Y. Chen and Y. Yang, "A new 4G architecture providing multimode terminals always best connected services," Wireless Communications, IEEE, vol. 14, pp. 36-41, 2007.

[6] P. Chiron, E. Njedjou, P. Seite, K. Gosse, E. Melin, and P. Roux, "Architectures for IP-based network-assisted mobility managemen across heterogeneous networks " Wireless Communications, IEEE, vol. 15, pp. 18-25, 2008.

[7] Moskowitz, R., Nikander, P.: Host Identity Protocol (HIP) Architecture. IETF, RFC 4423 (2006).

[8] Koundourakis, G., Axiotis, D.I., and Theologou, M.: Networkbased access selection in composite radio environments. In: IEEE Wireless Communications and Networking Conference 2007, Hong Kong (2007).

[9] Liu, X., Li, V. O. K., and Zhang, P.: NXG04-4: Joint radio resource management through vertical handoffs in $4 \mathrm{G}$ networks. In: IEEE Global Telecommunications Conference 2006, San Francisco (2006).

[10] Ormond, O., Perry, P., and Murphy, J.: Network selection decision in wireless heterogeneous networks. In: IEEE 16th International Symposium on Personal, Indoor and Mobile Radio Communications, Berlin (2005).

[11] Taha, A.-E.M., Hassanein, H.S. and Mouftah, H.T.: On robust allocation policies in wireless heterogeneous networks. In: First International Conference on Quality of Service in Heterogeneous Wired/Wireless Networks, Dallas (2004).

[12] B. Yang and P. Mohapatra, "DiffServ-aware multicasting Source", Transactions of the High Speed Networks Journal, Vol. 13, pp. 3757, January 2004

[13] R. Bless, K. Wehrle, "IP Multicast in Differentiated Services (DS) Networks", IETF RFC 3754, April 2004.

[14] R. Bless, K. Nichols and K. Wehrle, "A Lower Effort Per-Domain Behavior (PDB) for Differentiated Services", IETF RFC 3662, December 2003.

[15] A. Neto, E. Cerqueira, A. Rissato, E. Monteiro and P. Mendes, "A Resource Reservation Protocol Supporting QoS-aware Multicast Trees for Next Generation Networks", In Proc. of the 12th IEEE Symposium on Computers and Communications, Aveiro, Portugal, July 2007.

[16] 3GPP TS 23.246: 3rd Generation Partnership Project; MBMS; Architecture and functional description.

[17] D. Thaler et al., 2008. Automatic IP Multicast Without Explicit Tunnels (AMT), IETF, draft-ietf-mboned-auto-multicast-09.txt, June 2008.

[18] D. M. Moen, "Overview of Overlay Multicast Protocols", http://bacon.gmu.edu/XOM/pdfs/Multicast\%20Overview.pdf

[19] J. Jannotti et al., "Overcast: Reliable Multicasting with an Overlay Network", Proceedings of the Fourth Symposium on Operating Systems Design and Implementation, San Diego, October 2000, pp 197-212.

[20] Know, Y., 2006. P2MP Session Management Scheme using SIP in MPLS-based Next Generation Network", COIN-NGNCON 2006, pp.183-185, 9-13 July (2006).

[21] Oberle, K. Wahl, S. Sitek, A., 2007. Enhanced Methods for SIP based Session Mobility in a Converged Network, 16th IST Mobile and Wireless Communications Summit, (2007).

[22] B. Landfeldt, T. Larsson, Y. Ismailov, A. Seneviratne, "SLM, A Framework for Session Layer Mobility Management", in Proc. 
IEEE International Conference on Computer Communications and Networks, pages 452-456, Natick, Massachusetts, Oct 1999.

[23] R. Handorean et al., 2005. Context Aware Session Management for Services in Ad Hoc Networks, Services Computing, IEEE International Conference on Services Computing, (2005).

[24] R. Thomas, D. Friend, L. Dasilva, A. Mackenzie: Cognitive networks: adaptation and learning to achieve end-to-end performance objectives, IEEE Communications Magazine, December 2006.

[25] R. Thomas, L. DaSilva, and A. B. MacKenzie, "Cognitive networks." IEEE Intl. Symposium on New Frontiers in Dynamic Spectrum Access Networks, 2005.
[26] TISPAN - Telecoms \& Internet converged Services \& Protocols for Advanced Networks, http://www.etsi.org/tispan/

[27] MBMS - 3GPP TS 23.246: "Multimedia Broadcast/Multicast Service (MBMS): Architecture and functional description"

[28] DVB - Digital Video Broadcasting, http://www.dvb.org/

[29] H. Holbrook, B. Cain, "Source-Specific Multicast for IP", IETF RFC 4607, Aug 2006.

[30] IEEE P802.21/D14, Draft Standard for Local and Metropolitan Area Networks: Media Independent Handover Services, September 2008 